\title{
Heterotypic signaling between dermal fibroblasts and melanoma cells induces phenotypic plasticity and proteome rearrangement in malignant cells
}

\author{
Dayelle S. Pessotti ${ }^{\mathrm{a}}$, Débora Andrade-Silva ${ }^{\mathrm{b}}$, Solange M.T. Serrano ${ }^{\mathrm{b}}$, André Zelanis ${ }^{\mathrm{a}, *}$

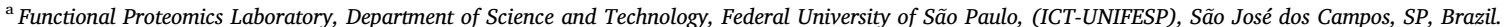 \\ ${ }^{\mathrm{b}}$ Laboratório de Toxinologia Aplicada, Center of Toxins, Immune-Response and Cell Signaling (CeTICS), Instituto Butantan, São Paulo, Brazil.
}

\section{A R T I C L E I N F O}

\section{Keywords:}

Heterotypic signaling

Melanoma

Fibroblast

Cancer-associated fibroblasts

Phenotypic plasticity

\begin{abstract}
A B S T R A C T
The signaling events triggered by soluble mediators released from both transformed and stromal cells shape the phenotype of tumoral cells and have significant implications in cancer development and progression. In this study we performed an in vitro heterotypic signaling assays by evaluating the proteome diversity of human dermal fibroblasts after stimulation with the conditioned media obtained from malignant melanoma cells. In addition, we also evaluated the changes in the proteome of melanoma cells after stimulation with their own conditioned media as well as with the conditioned medium from melanoma-stimulated fibroblasts. Our results revealed a clear rearrangement in the proteome of stromal and malignant cells upon crosstalk of soluble mediators. The main proteome signature of fibroblasts stimulated with melanoma conditioned medium was related to protein synthesis, which indicates that this process might be an early response of stromal cells. In addition, the conditioned medium derived from 'primed' stromal cells (melanoma-stimulated fibroblasts) was more effective in altering the functional phenotype (cell migration) of malignant cells than the conditioned medium from nonstimulated fibroblasts. Collectively, self- and cross-stimulation may play a key role in shaping the tumor microenvironment and enable tumoral cells to succeed in the process of melanoma progression and metastasis. Although the proteome landscape of cells participating in such a heterotypic signaling represents a snapshot of a highly dynamic state, understanding the diversity of proteins and enriched biological pathways resulting from stimulated cell states may allow for targeting specific cell regulatory motifs involved in melanoma progression and metastasis.
\end{abstract}

\section{Introduction}

The signaling events triggered by soluble mediators released from both transformed and stromal cells shape the phenotype of tumoral cells and have significant implications in cancer development and progression [1]. Hence, although autocrine secretion is an important source of oncogenic signals for tumoral cells, cancer progression and metastasis are strongly associated to the cellular crosstalk that exists among the distinct cell types within the tumoral microenvironment [2-4]. Such a heterotypic signaling has important implications for the malignancy of distinct cancer types and is related to the establishment of the pre-metastatic niche in distant sites [4]. Therefore, the comprehensive characterization, at the molecular level, of secreted factors from tumoral cells is of paramount importance in oncology research $[1,4-6]$. In this context, proteomic analysis of the secretomes obtained from stromal cells (fibroblasts) and metastatic melanoma cell lines allowed for the identification of signatures of protein expression and potential regulatory motifs which can eventually be targeted for therapeutic intervention in melanoma [7]. From the functional point of view, conditioned media/secretomes from tumoral cells have been extensively used as sources of oncogenic stimuli in distinct experimental models. Among the main biological implications of tumoral secreted factors are resistance and tumor progression in malignant cells [8], phenotypic shifts in cells subjected to tumoral stimuli [3], the emergence of metastatic features in stimulated cells, such as invasion and proliferation and others $[1,9,10]$.

Since the tumoral microenvironment encompasses several cell types, including stromal, primary tumoral and immune cells, the analysis of individual secreted factors together with their contribution to the cancer phenotype is challenging. Moreover, the dynamic crosstalk

\footnotetext{
* Corresponding author at: Functional Proteomics Laboratory, Department of Science and Technology, Federal University of São Paulo (ICT-UNIFESP), São José dos Campos, São Paulo, Brazil, Rua Talim, 330, 12231-280.

E-mail address: andre.zelanis@unifesp.br (A. Zelanis).
} 
that exists among the different cell types within the tumoral microenvironment leads to significant modulation of the corresponding signaling circuits that ultimately leads to cancer progression. A recurrent cell type present in tumoral stroma and surroundings are cancer-associated fibroblasts. These cancer-associated cells have distinct protein expression programs in comparison to their 'normal' counterparts and are strongly correlated to the main phenotypic switches found in cancer cells $[1,2,11]$. Therefore, the investigation of the ability of cancer cells to stimulate host fibroblasts and promoting a reactive stroma is of great importance for the understanding of altered biological pathways as well as for finding potential targets for therapeutic intervention.

In this respect, in this study we aimed to perform an in vitro heterotypic signaling assay, by evaluating the proteome diversity of human dermal fibroblasts after their stimulation with the conditioned media obtained from malignant melanoma cells. In addition, we also evaluated the changes in the proteome of melanoma cells after stimulation with their own conditioned media as well as with the conditioned medium from melanoma-stimulated fibroblasts. Our results underscore an expressive proteome rearrangement in stimulated cells, resulting in an enhanced secretory phenotype that might be associated to the phenotypic plasticity of tumoral cells upon heterotypic signaling.

\section{Material and methods}

\subsection{Cell culture and conditioned media harvesting}

Cell lines Hs 68 (CRL-1635 $5^{\mathrm{TM}}$ ), a dermal fibroblast from normal skin and A375 (CRL-1619 ${ }^{\mathrm{TM}}$ ), an epithelial cell derived from malignant melanoma from primary source, were obtained from the American Type Culture Collection (ATCC, USA). All cell lines were cultured in Dulbecco's Modified Eagle Medium (DMEM) containing $1.5 \mathrm{~g} / \mathrm{L}$ of sodium bicarbonate, $100 \mathrm{mg} / \mathrm{L}$ streptomycin, $25 \mathrm{mg} / \mathrm{L}$ ampicillin, $4 \mathrm{mM}$ Glutamine and $10 \%$ of Fetal Bovine Serum (FBS). Cells were grown in a humidified incubator at $37{ }^{\circ} \mathrm{C}$, with $5 \% \quad \mathrm{CO}_{2}$. The $3-(4,5-$ Dimethylthiazol-2-yl)-2,5-Diphenyltetrazolium Bromide assay (MTT) (Sigma, USA) was used for the evaluation of viability in cells subjected to the incubation with the different experimental treatments. Three conditioned media were used in this work, namely: (i) melanoma conditioned medium (MCM), which was obtained after culturing subconfluent A375 cells in T175 $\mathrm{cm}^{2}$ flasks for $24 \mathrm{~h}$, under phenol red-free and FBS-free DMEM; (ii) stimulated-fibroblast conditioned medium (sFCM), which was obtained after stimulating dermal fibroblasts (Hs68 cells) with $20 \%$ (vol/vol) of MCM in DMEM for $24 \mathrm{~h}$; this stimulus was removed and cells were re-incubated for $12 \mathrm{~h}$ with FBS-free DMEM when the sFCM was collected; and (iii) a conditioned medium obtained from dermal fibroblasts incubated for $24 \mathrm{~h}$ in FBS-free DEMEM (FCM). For all the experimental conditions, the conditioned media were centrifuged (390 $\mathrm{xg}, 3 \mathrm{~min}$, at room temperature) for removing any remaining cell, filtered $\left(0.22 \mu \mathrm{m}\right.$; Millipore, USA) and stored at $-80{ }^{\circ} \mathrm{C}$ until use.

\subsection{Cell treatments and lysis}

Subconfluent cell cultures (in $100 \mathrm{~mm}$-diameter culture dishes) were washed three-times with warm buffered phosphate-saline (PBS without $\mathrm{Ca}^{2+}$ and $\mathrm{Mg}^{2+}$ ) and subjected to the treatment with the three different conditioned media (MCM, sFCM or FCM) for $24 \mathrm{~h}$ in a humidified incubator at $37{ }^{\circ} \mathrm{C}$, with $5 \% \mathrm{CO}_{2}$. Briefly, to investigate the effects of melanoma conditioned medium (MCM) upon cell cultures, dermal fibroblasts and melanoma cells (Hs68 and A375 cell lines, respectively) were incubated with $20 \%$ (vol/vol) of MCM (in DMEM). In addition, the effects of the conditioned medium from MCM-stimulated fibroblasts were evaluated after incubating melanoma cells (A375 cell line) with either $20 \%$ (vol/vol) of sFCM or $20 \%$ (vol/vol) of FCM (in DMEM). After $24 \mathrm{~h}$, cultures were subjected to mechanical lysis with a cell scrapper and $1 \mathrm{~mL}$ of cell lysis buffer (2\% CHAPS, $150 \mathrm{mM} \mathrm{NaCl}$ in
HEPES $50 \mathrm{mM}, \mathrm{pH}$ 7.5) with the addition of a protease inhibitor cocktail (SIGMAFAST ${ }^{\mathrm{Tm}}$, Sigma, USA). Cell lysates were incubated in icebath for $30 \mathrm{~min}$ under mild agitation. After centrifugation $(14,000 \mathrm{xg}$, $10 \mathrm{~min}, 4^{\circ} \mathrm{C}$ ) supernatants were removed, subjected to protein quantitation using the Bradford method [12] and stored at $-80^{\circ} \mathrm{C}$ until use. Cell lysates were independently obtained from three biological replicates derived from each cell line. In summary, we evaluated the proteome profile of (i) dermal fibroblasts which were subjected to the treatment with the conditioned medium derived from melanoma cells, as well as the proteome profile of melanoma cells which were subjected to either (ii) its own conditioned medium or (iii) to the conditioned medium derived from melanoma-stimulated fibroblasts.

\subsection{In-solution trypsin digestion}

The in-solution trypsin digestion was performed as described earlier [7]. Briefly, a solution of $6 \mathrm{M}$ guanidine hydrochloride ( $\mathrm{GuHCl}$ ) was added to a sample of $100 \mu \mathrm{g}$ of protein from each cell lysate to a final concentration of $3 \mathrm{M} \mathrm{GuHCl}$, followed by the addition of $5 \mathrm{mM}$ dithiothreitol (DTT) (final concentration). The mixture was incubated at $37{ }^{\circ} \mathrm{C}$ for $1 \mathrm{~h}$. Iodoacetamide (IAA) was then added to a final concentration of $15 \mathrm{mM}$ and the samples were incubated for $30 \mathrm{~min}$ at room temperature, in the dark. To quench the excess of IAA, DTT was added to a final concentration of $15 \mathrm{mM}$ followed by the incubation of samples for $20 \mathrm{~min}$ at room temperature. Clean-up of samples was performed by the addition of ice-cold acetone ( 8 volumes) and methanol (1 volume), followed by the incubation of samples for $2 \mathrm{~h}$ at $-80{ }^{\circ} \mathrm{C}$. After centrifugation at $14,000 \mathrm{xg}$ for $10 \mathrm{~min}$, protein pellets were washed twice with one volume of ice cold methanol and then resolubilized with $\mathrm{NaOH}$ solution (final concentration of $10 \mathrm{mM}$ ), followed by the addition of HEPES buffer (final concentration of $50 \mathrm{mM}$ ), $\mathrm{pH} 7.5$, to a final volume of $100 \mu \mathrm{L}$. Trypsin (Proteomics grade; Sigma, USA) was added at $1: 100$ ratio (enzyme/substrate) and protein samples were incubated at $37{ }^{\circ} \mathrm{C}$ for $18 \mathrm{~h}$. Tryptic peptides were desalted using C18 StageTips [13], dried in a SpeedVac and resolubilized in $50 \mu \mathrm{L}$ of $0.1 \%$ formic acid prior to nanoflow liquid chromatography/tandem mass spectrometry (LC - MS/MS) analysis.

\subsection{Mass spectrometric analysis}

An aliquot ( $5 \mu \mathrm{L}$, corresponding to $3 \mu \mathrm{g}$ of peptides) of the resulting peptide mixture was injected into a trap column packed with $\mathrm{C} 18$ $(100 \mu \mathrm{m}$ i.d. $\times 2 \mathrm{~cm})$ for desalting with $100 \%$ solvent A $(0.1 \%$ formic acid). Peptides were then eluted onto an analytical column $(75 \mu \mathrm{m}$ i.d. $\times 100 \mathrm{~mm}$ ) packed in house with Aqua ${ }^{\circledR} \mathrm{C}-185 \mu \mathrm{m}$ beads (Phenomenex, USA). Nanoflow liquid chromatography was performed on an Easy nanoLC system (Thermo Fisher Scientific, USA) coupled to an LTQ-Orbitrap Velos mass spectrometer (Thermo Fisher Scientific, USA). Peptides were loaded onto the column with solvent A $(0.1 \%$ formic acid) and eluted within a $120 \mathrm{~min}$ chromatographic run, including a linear gradient from 4 to $30 \%$ of solvent B $(0.1 \%$ formic acid in acetonitrile) at a flow rate of $200 \mathrm{~nL} / \mathrm{min}$. Spray voltage was set at $2.1 \mathrm{kV}, 200{ }^{\circ} \mathrm{C}$ and the mass spectrometer was operated in data dependent mode, in which one full MS scan was acquired in the $m / z$ range of 400-1800 followed by MS/MS acquisition using Collision Induced Dissociation (CID) of the twelve most intense ions from the MS scan. MS spectra were acquired in the Orbitrap analyzer at 60,000 resolution (at $400 \mathrm{~m} / \mathrm{z}$ ). Dynamic exclusion was defined by a list size of 500 features and exclusion duration of $90 \mathrm{~s}$. For the survey (MS) scan Automatic Gain Control (AGC) target value of 1000,000 was set whereas the AGC target value for the fragment ion (MS/MS) spectra was set to 10,000 ions. The lower threshold for targeting precursor ions in the MS scans was 3000 counts. 
A

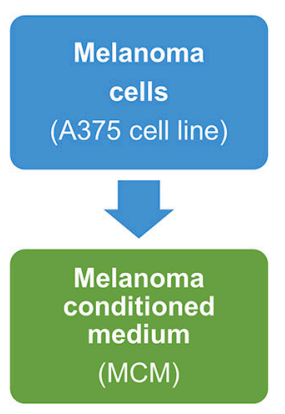

\section{Conditioned media}

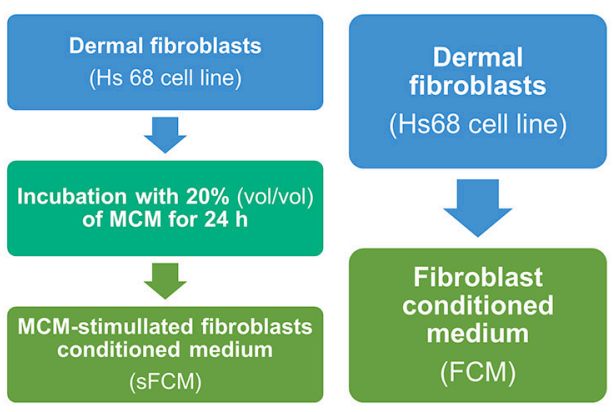

B

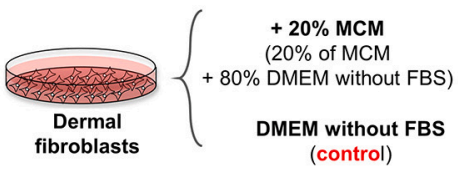

Experimental conditions

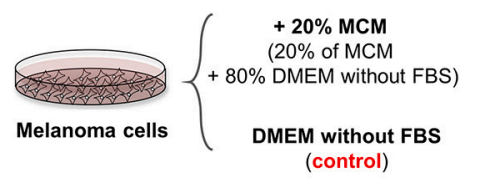

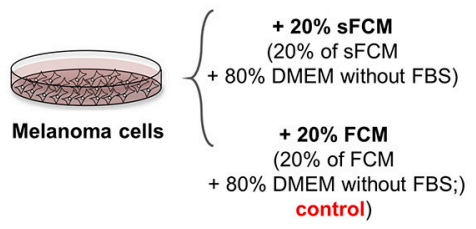

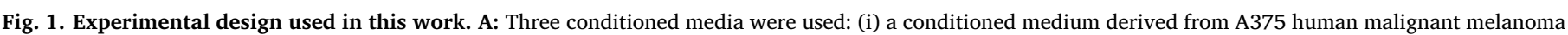

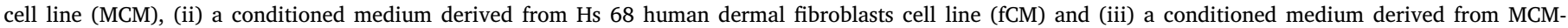

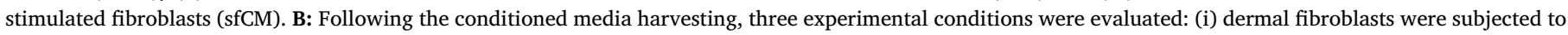

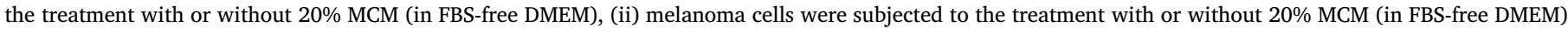

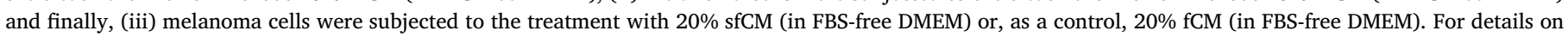
conditioned media preparation and experimental conditions, see Material and Methods section.

\subsection{Proteomics data processing}

Mass spectrometric (RAW) data were analyzed with MaxQuant software [14] (version 1.5.8.3). A False Discovery Rate (FDR) of 1\% was required for both protein and peptide-to-spectrum match identifications. LTQ-Orbitrap Velos raw data were searched against a target database restricted to the taxonomy 'Homo sapiens' (UniProt/SwissProt release 2019_06; 20,431 entries). This database was also combined with the sequences of 245 common contaminants and concatenated with the reversed versions of all sequences. Enzyme specificity was set to trypsin and up to two missed cleavages were allowed; cysteine carbamidomethylation was selected as fixed modification whereas methionine oxidation, glutamine/asparagine deamidation and protein $\mathrm{N}$-terminal acetylation were selected as variable modifications. Peptide identification was based on a search with an initial mass deviation of the precursor ion of $7 \mathrm{ppm}$ and the fragment mass tolerance was set to $0.5 \mathrm{Da}$. Label-free quantitation was performed using the MaxLFQ algorithm [15], with the 're-quantify' and 'match between runs' functions of MaxQuant software enabled. As is observed from complex proteomes such as those of vertebrates, peptides can be shared between homologous proteins or splice variants, leading to "protein groups". For each protein group in the MaxQuant's 'proteinGroups.txt' file, the first protein entry was selected as representative.

\subsection{Bioinformatic analyses}

Protein identifications were accepted if they contained at least two unique peptides. Only proteins identified/quantified in the three biological replicates were selected for further analysis. All the protein intensity values were log2-transformed and quantile-normalized using the 'preprocessCore' library in $\mathrm{R}$ scripting and statistical environment $[16,17]$. Statistical analyses were performed using the 'limma' package in R/Bioconductor [18,19]. After fitting a linear model to the data, an empirical Bayes moderated $t$-test was used for the comparisons and $p$ values were adjusted for multiple testing with the Benjamini-Hochberg method. Proteins with an adjusted p-value $\leq 0.05$ and $\log _{2}$ (fold change) $>1$ and $<-1$ were considered to be differentially expressed. Correlations between replicates were also calculated in $\mathrm{R}$, using Pearson correlation coefficient. Gene ontology enrichment analysis was carried out using STRING database [20] (https://string-db. org/); false discovery rates $\leq 0.05$ were considered. Protein interaction network was built after obtaining high-confidence interactions (i.e. interaction scores $\geq 0.7$ ) from the STRING database. The mass spectrometry proteomics data have been deposited to the ProteomeXchange Consortium via the PRIDE partner repository [21] with the dataset identifier PXD020426.

\subsection{Cell migration assay - transwell assay}

Melanoma cells (A375 cell line; $2 \times 10^{3}$ cells) were plated in the upper chambers of $6.5 \mathrm{~mm}$-diameter, $8 \mu \mathrm{m}$ pore transwells (24-wells plate, Corning, USA) after a starvation period of $24 \mathrm{~h}$. Transwell plates were placed in a humidified incubator at $37{ }^{\circ} \mathrm{C}$, with $5 \% \mathrm{CO}_{2}$ and the cells were allowed to migrate toward the lower chamber containing distinct chemoattractancts, namely: 20\% sFCM, 20\% FCM, DMEM containing 10\% FBS (positive control) or DMEM without FBS (negative control). After $24 \mathrm{~h}$ of incubation, cells at the top chamber were removed using a cotton swab and the cells at the bottom of the insert filter were fixed with $3.7 \%$ formaldehyde for $2 \mathrm{~min}$ and incubated in absolute methanol for $20 \mathrm{~min}$ at room temperature. After washing the inserts twice with PBS, cells were stained after the addition of a $0.2 \%$ violet crystal in $20 \%$ ethanol solution. The inserts were incubated in a $1 \%$ SDS solution for the elution of the dye and the absorbance was measured at $570 \mathrm{~nm}$ in a microplate reader. Cell migration was expressed as percentage of migration normalized in relation to the negative control condition. Experiments were performed in triplicate for each treatment. Statistical analysis was carried out using ANOVA with GraphPad Prism 7.0 software; $p$-values $\leq 0.05$ were considered to be significant. 
Table 1

Protein content $(\mathrm{mg} / \mathrm{mL})$ of cell lysates after the treatment with or without melanoma conditioned medium (MCM).

\begin{tabular}{llll}
\hline Cell line & Without MCM & With MCM & $p$-value (t-test) \\
\hline Hs68 & $0.13 \pm 0.01$ & $0.17 \pm 0.03$ & 0.2044 \\
A375 & $1.05 \pm 0.09$ & $1.27 \pm 0.04$ & 0.0757 \\
\hline
\end{tabular}

\section{Results and discussion}

\subsection{Melanoma conditioned medium (MCM) promotes a proteome rearrangement in dermal fibroblasts and in malignant melanoma cells}

The experimental design used in this work allowed us to profile both qualitative and quantitative differences in cell line proteomes after their stimulation with distinct conditioned media derived from tumoral cells. Fig. 1 depicts the process of conditioned media production as well as the experimental treatments that were used in this work. Cell viability was $\geq 90 \%$ upon conditioned medium harvesting as evaluated by trypan blue analysis (data not shown). However, although not statistically significant we observed that cell viability (measured by the MTT assay) decreased after the treatment with increasing volumes of conditioned media (Supplementary fig. 1). Therefore, all the experiments with the different conditioned media were performed by incubating cells with $20 \%$ (vol/vol) of MCM.

Both dermal fibroblasts and melanoma cells stimulated by MCM displayed a slightly higher (although not statistically significant) protein content (Table 1). Overall, when analyzing the proteome of dermal fibroblast lysates with or without stimulation with MCM we identified 1078 proteins (Supplementary table 1; for metadata on protein identification please refer to Supplementary table 2), with Pearson's correlation $\geq 0.9$ among the three biological replicates (Supplementary fig. 2).

The analysis of the proteome from the dermal fibroblasts stimulated with MCM or with FBS-free DMEM (control condition) revealed that most of the identified proteins are shared by these conditions (Fig. 2A). However, a higher number of exclusive proteins were identified in the MCM-treated condition. Therefore, the MCM-treatment of dermal fibroblasts increased the proteome diversity and promoted a slight increase in the protein content of stimulated cells. A gene ontology (GO biological process) analysis was performed upon the exclusive proteins in both conditions and revealed an enrichment in proteins related to mRNA catabolic process, protein targeting to membrane and translation initiation in the proteome of fibroblasts after their stimulation with MCM (Fig. 2B). No significant GO enrichment was found for the 23 exclusive proteins identified in fibroblasts stimulated with FBS-free DMEM (i.e. without MCM; control condition). It is important to note that exclusive proteins in our comparisons displayed high correlation among biological replicates and, more importantly, were identified by both MS1 and MS/MS signals that were absent in the counterpart.

Growth factors such as Hepatoma-derived growth factor (HDGF) and Neurosecretory protein VGF were among the exclusive proteins identified in the fibroblasts treated with MCM. The up-regulation of HDGF has been implicated in cancer progression and is frequently associated to the enhanced secretory phenotype of cancer-associated fibroblasts (CAFs) [22,23]. Neurosecretory protein VGF activates prosurvival signaling pathways such as PI3K/AKT/mTOR and MAPK/ ERK1/2 and was recently shown to be overexpressed in prostate cancer patients with early relapse [24]. Protein kinases such as dual specificity mitogen-activated protein kinase kinase 1(MAPKK 1), Glycogen synthase kinase-3 beta (GSK3B) and Focal adhesion kinase 1 (PTK2) were also identified exclusively in fibroblasts treated with MCM. All these three proteins are involved in malignant transformation, with main roles in tumorigenic process, including cell survivor, epithelial-mesenchymal transition (EMT) and cell migration. MAPKK1 is involved in
BRAF activation and, depending on the cellular context, the downstream activated pathway may lead to diverse biological functions including cell growth, adhesion, survival and differentiation [25]. Zhou and coworkers showed that the interaction of GSK3B with the E-cadherin transcriptional repressor, Snail protein, functions as a molecular switch for signaling pathways that eventually lead to EMT [26]. Focal adhesion kinase 1 displays an essential role in regulating cell migration, adhesion and spreading, mainly through the integrin $\beta 1$-FAK signaling pathway, and its overexpression may play a role in metastasis [2,27] Indeed, conditioned medium from oral squamous cell carcinoma CAFs displaying high levels of PTK2 induced invasion and migration of squamous SCC-25 cells [28].

We also performed statistical analysis ( $t$-test) upon the shared proteins in order to verify differentially expressed proteins between the two experimental conditions. Thirty-two proteins were found to be upregulated in fibroblast cells without the addition of MCM in comparison to 105 up-regulated proteins that were found after MCM stimulation (Fig. 2C; Supplementary table 3). Interestingly, as also verified in the set of exclusive proteins from MCM-stimulated fibroblasts, we observed an up-regulation of proteins related to protein/mRNA metabolism in (Fig. 2D), suggesting that MCM triggered biological pathways related to protein synthesis. On the other hand, proteins mainly related to redox homeostasis and cysteine peptidase-mediated apoptotic processes where up-regulated in the fibroblasts with no stimulation with MCM.

Autocrine effects of MCM were evaluated by treating melanoma cells with their own conditioned medium. Although most of the identified proteins (1473; Supplementary table 1) were shared between the two experimental conditions, 79 proteins were identified exclusively in melanoma cells stimulated with FBS-free DMEM (i.e. without MCM; control condition) whereas 54 proteins were found to be exclusive after treating melanoma cells with MCM (Fig. 3A). While no significant enrichment was observed for GO terms (biological process) in the exclusive proteins identified in melanoma cells without MCM, catabolic processes and protein folding were enriched in the set of exclusive proteins in melanoma cells subjected MCM treatment (Fig. 3B). Statistical comparison ( $t$-test) between the two conditions revealed a small set of differentially expressed proteins; among which, Huntingtin-interacting protein $\mathrm{K}$ (HYPK; $\log _{2}$ (fold change) $=-1.8$ ), Dynactin subunit 5 (DCTN5; $\log _{2}$ (fold change) $=-1.2$ ) and Ribonucleoside-diphosphate reductase subunit $2\left(\mathrm{RRM} 2 ; \log _{2}\right.$ (fold change $\left.)=-2.0\right)$ were found to be up-regulated in melanoma cells treated with MCM whereas E3 ubiquitin-protein ligase (TRIM56, $\log _{2}$ (fold change) $=7.9$ ) and Larelated protein 4 (LARP4; $\log _{2}$ (fold change $)=1.6$ ) were up-regulated in the control condition (Fig. 3C; Supplementary table 4). Although no significant biological process was found to be enriched in differentially expressed proteins from either of the experimental conditions, it is reasonable to suggest that cancer-related processes might be benefited from the up-regulation of proteins expressed after MCM treatment in melanoma cells. For example, HYPK protein has a chaperone-like activity, preventing polyglutamate aggregation of Huntingtin protein and is a negative regulator of apoptotic process in neuroblasts [29]. Dynactin is large protein complex which interacts and is required for proper dynein functions [30]. The Dynein/Dynactin complex has a main role in orienting the cell division plane and disruptions in mitotic spindle orientation may eventually be related to the overgrowth of epithelial cells [31]. Ribonucleoside-diphosphate reductase subunit M2 (RRM2) belongs to ribonucleotide reductase complex and is involved in the pathway of DNA replication, providing the precursors necessary for DNA synthesis [32]. In fact, ribonucleoside-diphosphate reductase subunit M2 is up-regulated in melanoma cells harboring BRAF ${ }^{\mathrm{V} 600 \mathrm{E}}$ mutation and such overexpression has been correlated with worse overall survival in melanoma patients; therefore this subunit might be targeted for combinatorial therapy in melanoma treatment $[33,34]$. 
A

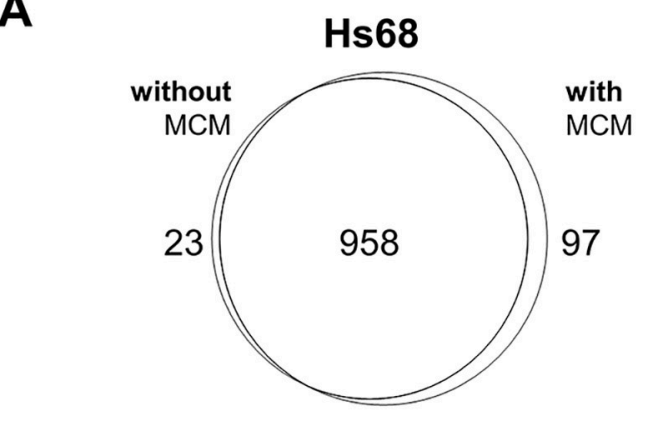

B

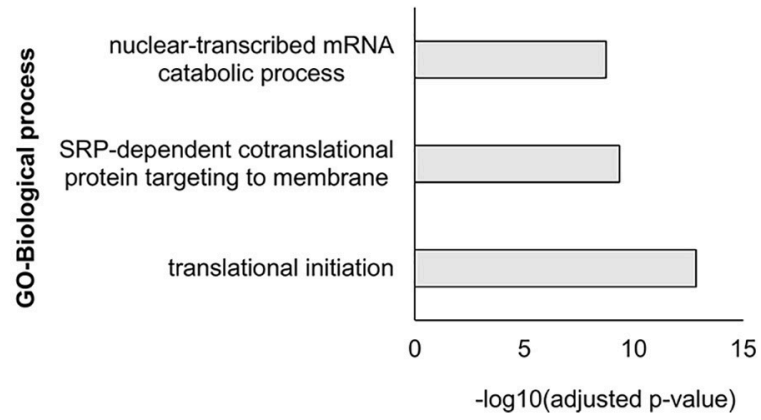

C

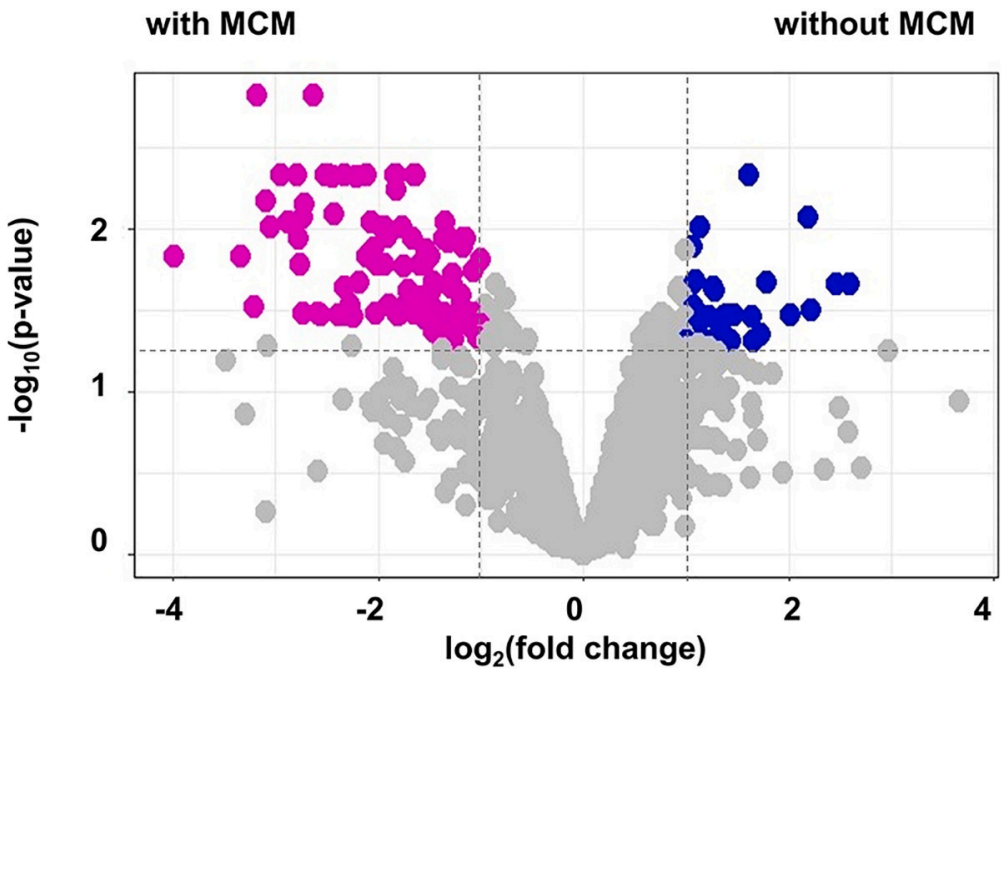

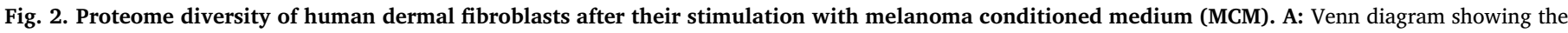

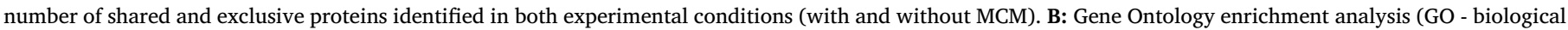

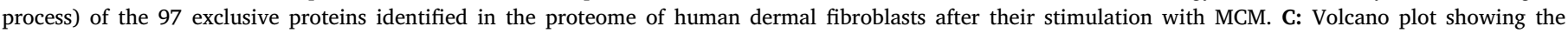

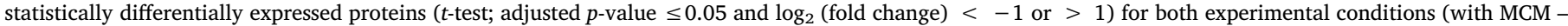

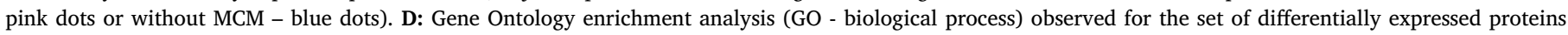
observed in (C).

A

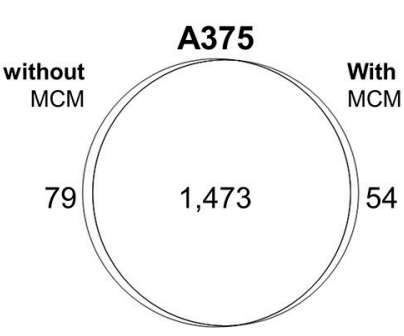

B

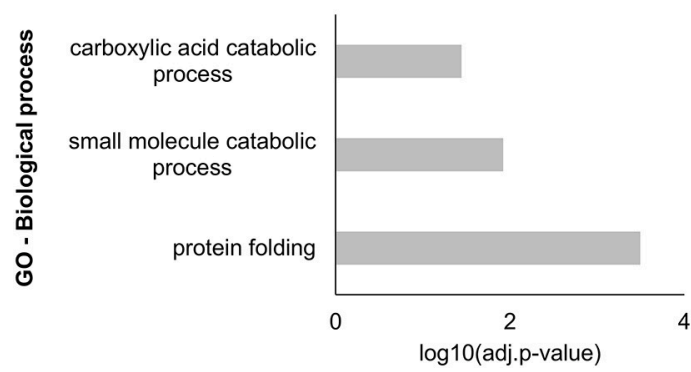

C

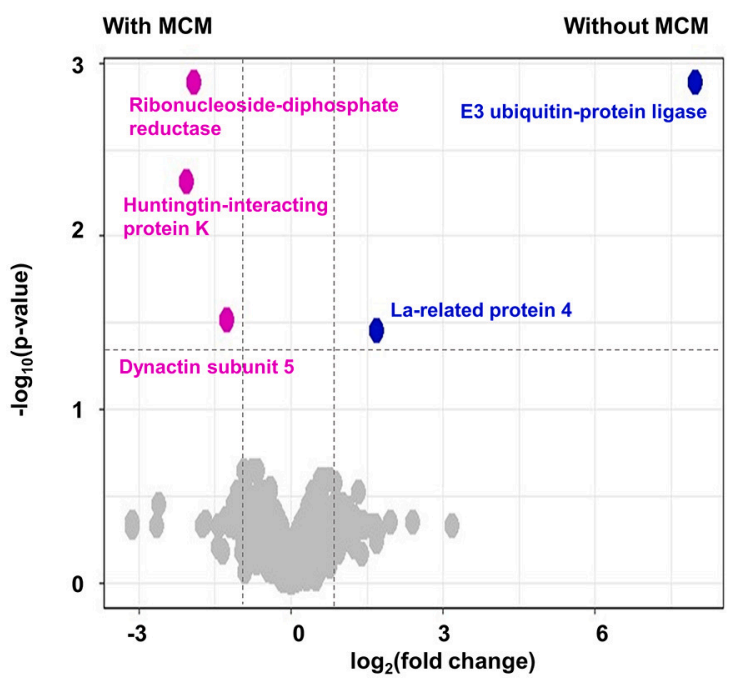

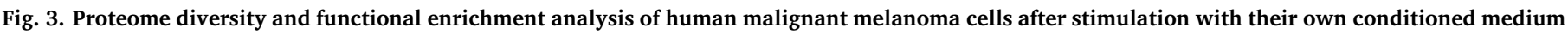

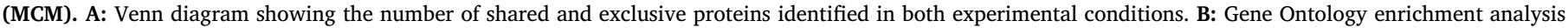

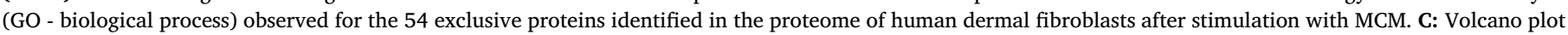

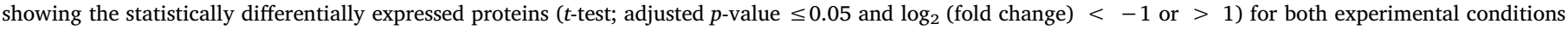
(with MCM - pink dots or without MCM - blue dots). 


\section{A}

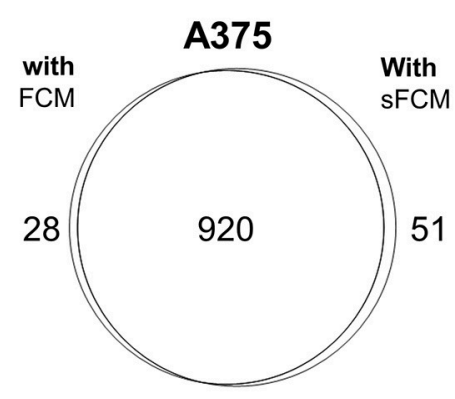

B
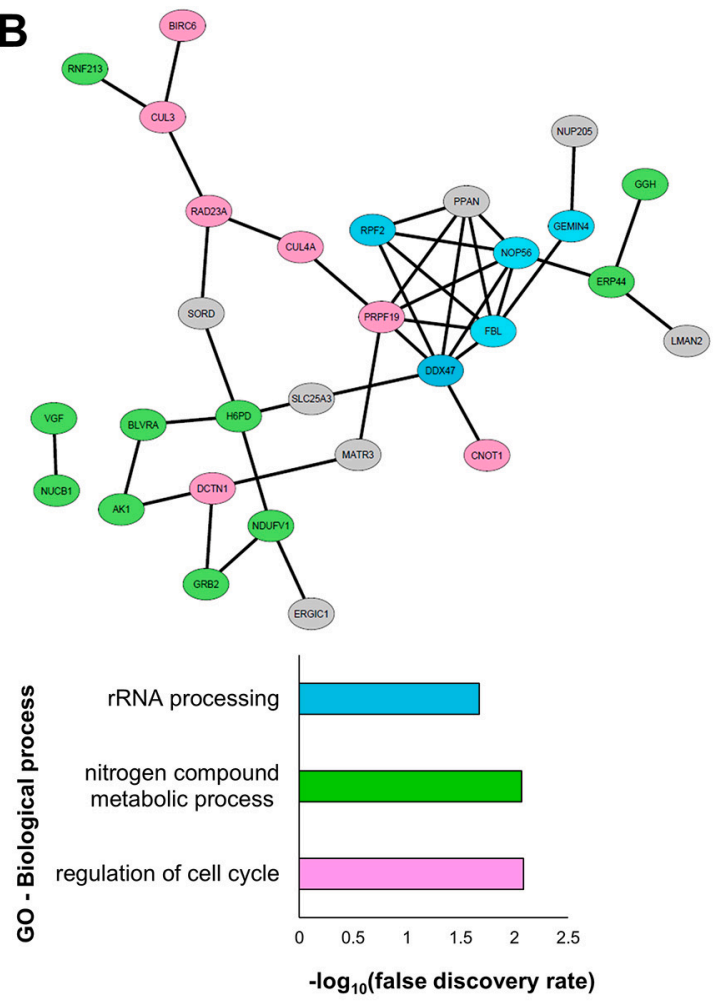

D
C

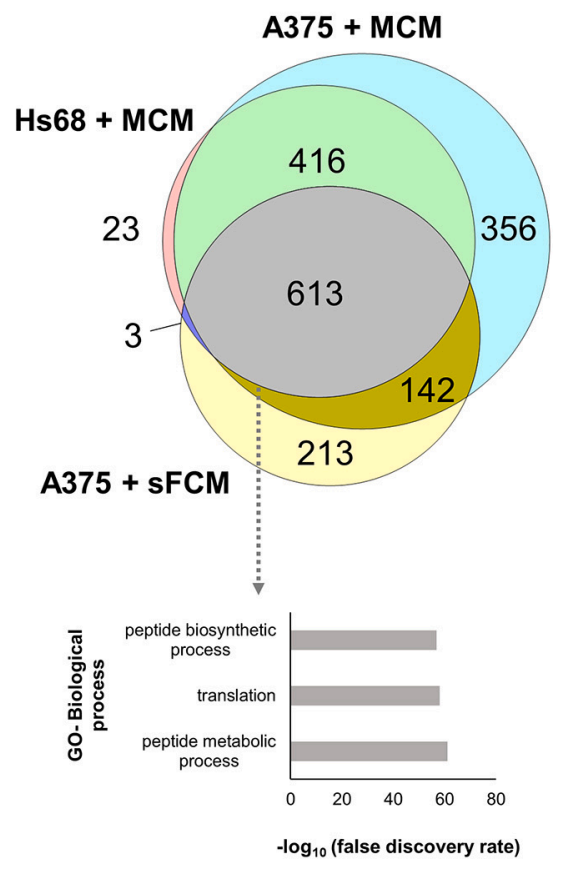

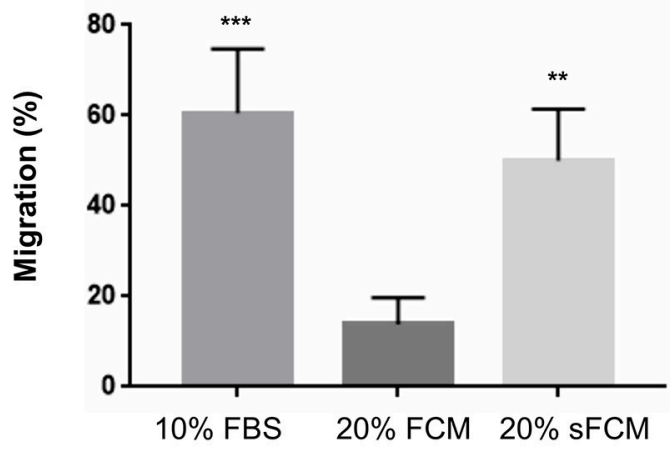

Treatments

Fig. 4. Proteome diversity, functional enrichment analysis and phenotypic plasticity of human malignant melanoma cells after the treatment with fibroblast-stimulated conditioned medium (sFCM). A: Venn diagram showing the number of shared and exclusive proteins identified in both experimental conditions. B: protein-protein interaction network derived from the set of exclusive proteins found in sFCM condition (upper panel) revealed the enrichment of biological processes related to the regulation of cell cycle and nitrogen metabolism (lower panel). Colored ellipses correspond to proteins associated to the enriched biological processes displayed in the bar chart (for further details, see Material and Methods section). C: Venn diagram showing the number of shared and exclusive proteins identified in all three experimental conditions evaluated in this work. The 613 overlapping proteins were evaluated for the functional enrichment analysis (GO-Biological process). D: transwell assay showing the migration of melanoma cells after stimulation with 10\% FBS (positive control), fibroblast conditioned medium (FCM) and MCM-stimulated fibroblasts ( $\mathrm{FFCM}$ ). Results were expressed as mean \pm standard deviation derived from three biological replicates. $(* * p<0,01$ and $* * * \mathrm{p}<0,001 ;$ ANOVA $)$.

\subsection{Stimulated fibroblast conditioned medium ( $S F C M$ ) altered the proteome of melanoma cells and enhanced their migration}

Our results illustrated a proteome rearrangement in MCM-stimulated fibroblasts which eventually enable a cancer-secretory phenotype in such cells, a recurrent feature observed in cancer-associated fibroblasts [2]. We reasoned whether such altered secretory phenotype in the conditioned medium of stimulated fibroblasts would be able to promote changes in the proteome of melanoma cells. From the biological point of view, this experimental approach was expected to somehow emulate part of the heterotypic signaling observed between cancer-associated fibroblasts (CAFs) and the tumoral cells within the tumoral microenvironment. We identified nearly 1000 proteins, with 51 proteins being exclusively identified after the stimulation of melanoma cells with the conditioned medium derived from MCM-stimulated fibroblasts $(\mathrm{sFCM})$ and 28 proteins exclusively identified after 
stimulating melanoma cells with the medium derived from 'nonprimed' fibroblasts (FCM; control condition) (Fig. 4A; Supplementary table 5). The analysis of interaction networks of the 51 exclusive proteins found in melanoma cells after the treatment with sFCM revealed clusters of functionally related proteins, in which processes associated with the metabolism of nitrogenated compounds, rRNA processing and regulating cell cycle were found to be enriched (Fig. 4B). No biological processes were found to be enriched in the set of the 28 proteins exclusively detected in the melanoma cells treated with FCM.

Interestingly, we found some overlap among the three experimental conditions that were evaluated in this study (i.e. MCM-stimulated fibroblasts, MCM-stimulated melanoma cells and sFCM-stimulated melanoma cells) (Fig. 4C). This was the case for 613 proteins which were identified in all three conditions. Again, biological processes related to protein metabolism, (translation, peptide biosynthetic processes, peptide metabolic process) were enriched in this group of overlapping proteins. Such overlap could suggest that MCM may invoke similar expression programs in both stromal and tumoral cells, which eventually lead to increased protein biosynthesis. We did not find any differentially expressed proteins after statistical analysis ( $t$-test) on the group of shared proteins between both conditions (melanoma cells with/without sFCM; data not shown). However, in order to evaluate whether the changes in the proteome diversity after the incubation of melanoma cells with sFCM translates into distinct functional phenotypes, we evaluated the chemotactic potential of sFCM in promoting melanoma cell migration, a recurrent feature of metastatic melanoma cells. Transwell assay showed that sFCM was able to promote cell migration, being significantly different when compared to the migration promoted by the fibroblast conditioned medium (FCM) upon melanoma cells (Fig. 4D). Interestingly, MCM was not able to promote the migration of both melanoma and fibroblasts cells in the transwell assay (data not shown).

\subsection{Phenotypic plasticity in melanoma upon heterotypic signaling}

Tumoral tissue is a composite of distinct cell types, with diverse gene expression programs and with some redundancy in the observed proteome. In this context, the phenotypic plasticity in gene expression programs of tumoral and stromal cells enables the viability of tumoral cells which might eventually outperforms the adversities found by rapid growing cells in normal tissues. Overall, our results pointed out to a significant rearrangement in the proteome of stromal (dermal fibroblasts) and malignant cells (melanoma cells) upon crosstalk of soluble mediators (Fig. 5).

The overlapping of identified proteins related to biosynthetic pathways in the distinct experimental conditions indicates that in our cellular model protein synthesis might be an early response of stimulated cells. In fact, the main proteome signature of stimulated cells was related to protein synthesis - the conditioned media from melanoma cells was able to set up an enhanced secretory phenotype in both (normal) dermal fibroblasts and melanoma cells, primarily by the upregulation of proteins related to protein biosynthetic pathways. This phenotypic switch mirrors the secretory phenotype found in CAFs and may have been triggered by the up-regulation of proteins related to biosynthetic processes, such as translation elongation and initiation factors, tRNA ligases, and ribosomal proteins, as described in Supplementary table 6. In fact, in the context of tumorigenesis, where altered cell signaling circuits enable a rapid growing of transformed cells, such an enhanced secretory phenotype may sustain this phenotypic switch. This might be the case for A375 melanoma cells, which display a characteristic V600E mutation in the cell membrane serine/ threonine-protein kinase B-Raf (BRAF), resulting in an enhanced transduction of mitogenic signals. Moreover, proteins related to cell adhesion and migration were also found to be upregulated in the proteome of stimulated cells. Li and coworkers showed that the invasive potential of human melanoma cell lines correlates with their ability to alter fibroblast gene expression in vitro [10]. According to the authors, the invasive potential of melanoma cells was enhanced after their coculture with stromal cells (dermal fibroblasts). In our results, although some migration had been found after the incubation of melanoma cells with fibroblast conditioned medium (FCM), this behavior was significantly enhanced by the conditioned medium derived from stimulated fibroblasts. Therefore, our results suggest that the conditioned medium derived from these 'primed' stromal cells (MCM-stimulated fibroblasts) was more effective in altering the functional phenotype of malignant cells than the fibroblast conditioned medium alone. Collectively, self- and cross-stimulation may play a key role in shaping the tumor microenvironment and, more importantly, enable tumoral cells to succeed in the process of melanoma progression and metastasis. It is important to emphasize, however, that given the high molecular/phenotypic heterogeneity found in melanoma, our cellular model and experimental workflow lack a more comprehensive set of molecular signals and the corresponding biological responses found in a complex environment since it was limited to using conditioned media derived from two cell lines. In this regard, further studies using distinct melanoma cell lines could address such limitations.

\section{Conclusions}

The imbalance caused by somatic mutational processes together with altered signaling pathways are recurrent features of many types of cancer. In this context, the crucial steps for cancer development and progression, such as the metastatic process, strongly rely on the crosstalk of soluble mediators within the tumoral microenvironment. The molecular and cellular components involved in pre-metastatic niche formation are mainly primary tumor-derived components, tumor-

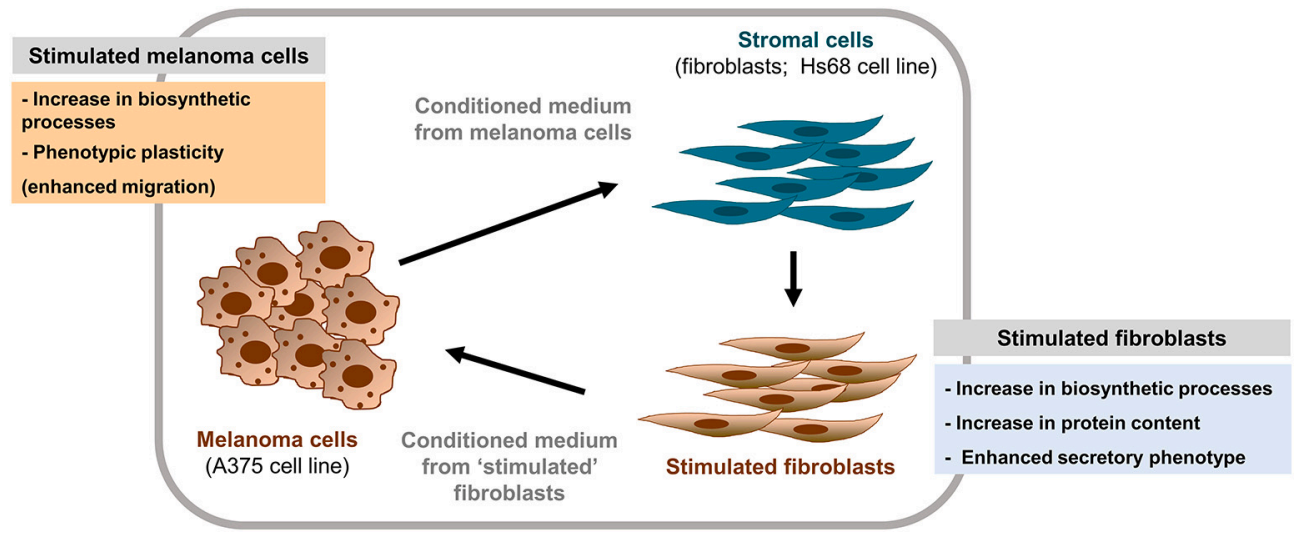

Fig. 5. Overview of the main functional implications of heterotypic signaling evaluated in this study. 
mobilized bone marrow-derived cells and the local stromal microenvironment of the host [1]. Therefore, since primary tumors can synthesize and secrete soluble mediators which ultimately modulate the microenvironment of distant sites, preparing them for tumor cell arriving, the identification of proteome alterations upon heterotypic signaling reported in this work shed light on some of the biological responses to the molecular interplay of effectors in a controlled experimental situation (i.e. under cell culture conditions). Whether this scenario is observed in a more complex environment such as the tumoral tissue remains to be further investigated. Nevertheless, our results suggest that the enhanced secretory phenotype likely enabled by increased protein synthesis may be a prominent feature in stimulated cells. In addition, we found that the chemotactic potential of the melanoma-stimulated fibroblast conditioned medium was more effective in promoting the migration of melanoma cells and such feature correlated with the up-regulation of proteins related to cell adhesion and migration in these stimulated cells.

Our experimental approach allowed for the identification of significantly altered biological pathways whose participating proteins might be further investigated for their mechanisms of signal transduction between fibroblasts and melanoma cells. In addition, in our cellular model, soluble mediators from tumoral cells seem to be predominantly involved in the crosstalk with stromal cells rather than in autocrine signaling. Therefore, under the experimental conditions described herein, once stimulated by tumoral effectors dermal fibroblasts might trigger pro-tumoral signaling events that ultimately would enable melanoma cells to quantitatively and qualitatively modify their secretory phenotype as well as their invasiveness. The main proteome signature resulting from our experiments was related to protein synthesis, including translation initiation, mRNA turnover and protein targeting. Therefore, although the main findings reported in this work need to be further explored by evaluating a more diverse experimental setup comprising distinct melanoma and stromal cell lines, proteins related to biosynthetic processes are likely to be drivers of the enhanced secretory phenotype of these 'primed' cells and should be further investigated.

Although the proteome landscape of cells participating in such a heterotypic signaling represents a snapshot of a highly dynamic state, understanding the diversity of proteins and enriched biological pathways resulting from stimulated cell states may allow for targeting specific cell regulatory motifs involved in melanoma progression and metastasis.

\section{Author contributions}

Conceived and designed the experiments: AZ and D.S.P; Performed the experiments: D.S.P and DAS. Analyzed the data: AZ and D.S.P; Writing the manuscript: AZ, D.S.P, DAS and S.M.T.S. Funding acquisition: AZ.

\section{Declaration of Competing Interest}

None.

\section{Acknowledgments}

This work was supported by grants from Fundação de Amparo à Pesquisa do Estado de São Paulo (FAPESP, grants \# 2014/06579-3, 2013/07467-1, 2016/16935-7, 2019/07282-8).

Credit author statement.

Conceived and designed the experiments: AZ and D.S.O.P; Performed the experiments: D.S.O.P and DAS. Analyzed the data: AZ and D.S.O.P; Writing the manuscript: AZ, D.S.OPP, DAS and S.M.T.S. Funding acquisition: AZ.

Declaration of Competing Interest.

The authors declare no competing interest.

\section{Appendix A. Supplementary data}

Supplementary data to this article can be found online at https:// doi.org/10.1016/j.bbapap.2020.140525.

\section{References}

[1] Y. Liu, X. Cao, Characteristics and significance of the pre-metastatic niche, Cancer Cell 30 (2016) 668-681.

[2] R. Kalluri, The biology and function of fibroblasts in cancer, Nat. Rev. Cancer 16 (2016) 582-598.

[3] A.I. Oliveira, S.I. Anjo, J. Vieira De Castro, S.C. Serra, A.J. Salgado, B. Manadas, B.M. Costa, Crosstalk between glial and glioblastoma cells triggers the "go-or-grow" phenotype of tumor cells, cell Commun, Signal. (2017) 15.

[4] R.N. Kaplan, R.D. Riba, S. Zacharoulis, H. Anna, L. Vincent, C. Costa, D.D. Macdonald, D.K. Jin, S.A. Kerns, Z. Zhu, D. Hicklin, Y. Wu, J.L. Port, E.R. Port, D. Ruggero, S.V. Shmelkov, K.K. Jensen, S. Rafii, D. Lyden, VEGFR1-positive haematopoietic bone marrow progenitors initiate the pre-metastatic niche, Nature 438 (2005) 820-827.

[5] M.H. Dias, E.S. Kitano, A. Zelanis, L.K. Iwai, Proteomics and drug discovery in cancer, Drug Discov. Today 21 (2016) 264-277.

[6] G.S. Karagiannis, M.P. Pavlou, E.P. Diamandis, Cancer secretomics reveal pathophysiological pathways in cancer molecular oncology, Elsevier B.V 4 (6) (2010) 496-510.

[7] T. Liberato, D.S. Pessotti, I. Fukushima, E.S. Kitano, S.M.T. Serrano, A. Zelanis, Signatures of protein expression revealed by secretome analyses of cancer associated fibroblasts and melanoma cell lines, J. Proteome 174 (2018) 1-8.

[8] A.C. Obenauf, Y. Zou, A.L. Ji, S. Vanharanta, W. Shu, H. Shi, X. Kong, M.C. Bosenberg, T. Wiesner, N. Rosen, R.S. Lo, J. Massagué, Therapy-induced tumour secretomes promote resistance and tumour progression, Nature 520 (2015) $368-372$.

[9] B. Izar, C.E. Joyce, S. Goff, N.L. Cho, P.M. Shah, G. Sharma, J. Li, N. Ibrahim, J. Gold, F.S. Hodi, L.A. Garraway, C.D. Novina, M.M. Bertagnolli, C.H. Yoon, Bidirectional cross talk between patient-derived melanoma and cancer-associated fibroblasts promotes invasion and proliferation, Pigment Cell Melanoma Res. 29 (2016) 656-668.

[10] L. Li, B. Dragulev, P. Zigrino, C. Mauch, J.W. Fox, The invasive potential of human melanoma cell lines correlates with their ability to alter fibroblast gene expression in vitro and the stromal microenvironment in vivo, Int. J. Cancer 125 (2009) 1796-1804.

[11] S. Madar, I. Goldstein, V. Rotter, "Cancer associated fibroblasts" - more than meets the eye, Trends Mol. Med. 19 (2013) 447-453.

[12] M.M. Bradford, A rapid and sensitive method for the quantitation of microgram quantities of protein utilizing the principle of protein-dye binding, Anal. Biochem. 72 (1976) 248-254.

[13] J. Rappsilber, M. Mann, Y. Ishihama, Protocol for micro-purification, enrichment, pre-fractionation and storage of peptides for proteomics using StageTips, Nat. Protoc. 2 (2007) 1896-1906.

[14] J. Cox, M. Mann, MaxQuant enables high peptide identification rates, individualized p.p.b-range mass accuracies and proteome-wide protein quantification, Nat. Biotechnol. 26 (2008) 1367-1372.

[15] J. Cox, M.Y. Hein, C.A. Luber, I. Paron, N. Nagaraj, M. Mann, Accurate proteomewide label-free quantification by delayed normalization and maximal peptide ratio extraction, termed MaxLFQ, Mol. Cell. Proteomics 13 (2014) 2513-2526.

[16] B.M. Bolstad, R.A. Irizarry, M. Astrand, T.P. Speed, A comparison of normalization methods for high density oligonucleotide array data based on variance and bias, Bioinformatics 19 (2003) 185-193.

[17] I. Ross, G. Robert, R. Thaka, R. Gentleman, R: a language for data analysis and graphics, J. Comput. Graph. Stat. 5 (1996) 299-314.

[18] R. Gentleman, V. Carey, D. Bates, B. Bolstad, M. Dettling, S. Dudoit, B. Ellis L. Gautier, Y. Ge, J. Gentry, K. Hornik, T. Hothorn, W. Huber, S. Iacus, R. Irizarry, F. Leisch, C. Li, M. Maechler, A. Rossini, G. Sawitzki, et al., Bioconductor: open software development for computational biology and bioinformatics, Genome Biol. 5 (2004) R80.

[19] G.K. Smyth, Linear models and empirical Bayes methods for assessing differential expression in microarray experiments linear models and empirical Bayes methods for assessing differential expression in microarray experiments, Stat. Appl. Genet. Mol. Biol. 3 (2004) 1-26.

[20] D. Szklarczyk, J.H. Morris, H. Cook, M. Kuhn, S. Wyder, M. Simonovic, A. Santos, N. T. Doncheva, A. Roth, P. Bork, L.J. Jensen, C. Von Mering, The STRING database in 2017: quality-controlled protein-protein association networks, made broadly accessible, Nucleic Acids Res., 45 (2017) D362-D368.

[21] J.A.J. Vizcaíno, E.E.W. Deutsch, R. Wang, A. Csordas, F. Reisinger, D. Ríos, J.A Dianes, Z. Sun, T. Farrah, N. Bandeira, P.A. Binz, I. Xenarios, M. Eisenacher, G. Mayer, L. Gatto, A. Campos, R.J. Chalkley, H.J. Kraus, J.P. Albar, S. MartinezBartolomé, et al., ProteomeXchange provides globally coordinated proteomics data submission and dissemination, 2014

[22] C.H. Bao, X.T. Wang, W. Ma, N.N. Wang, E. Un Nesa, J.B. Wang, C. Wang, Y. Bin Jia, K. Wang, H. Tian, Y.F. Cheng, Irradiated fibroblasts promote epithelial - mesenchymal transition and HDGF expression of esophageal squamous cell carcinoma, Biochem. Biophys. Res. Commun. 458 (2015) 441-447.

[23] A. Matsuyama, H. Inoue, K. Shibuta, Y. Tanaka, G.F. Barnard, K. Sugimachi, M. Mori, Hepatoma-derived growth factor is associated with reduced sensitivity to irradiation in esophageal cancer, Cancer Res. 61 (2001) 5714-5717. 
[24] M. Seifert, C. Peitzsch, I. Gorodetska, C. Börner, B. Klink, A. Dubrovska, Networkbased analysis of prostate cancer cell lines reveals novel marker gene candidates associated with radioresistance and patient relapse, PLoS Comput. Biol. 15 (2019).

[25] X. Liu, S. Yan, T. Zhou, Y. Terada, R.L. Erikson, The MAP kinase pathway is required for entry into mitosis and cell survival, Oncogene 23 (2004) 763-776.

[26] B.P. Zhou, J. Deng, W. Xia, J. Xu, Y.M. Li, M. Gunduz, M.C. Hung, Dual regulation of snail by GSK-3ß-mediated phosphorylation in control of epithelial-mesenchymal transition, Nat. Cell Biol. 6 (2004) 931-940.

[27] X. Wang, Q. Zhou, Z. Yu, X. Wu, X. Chen, J. Li, C. Li, M. Yan, Z. Zhu, B. Liu, L. Su, Cancer-associated fibroblast-derived Lumican promotes gastric cancer progression via the integrin $\beta 1-F A K$ signaling pathway, Int. J. Cancer 141 (2017) 998-1010.

[28] Z.T. and T.S. Anjie Min, Chao Zhu, Jingyi Wang, Shuping Peng, Cijun Shuai, Shan Gao, Focal Adhesion Kinase Knockdown in Carcinoma-Associated Fibroblasts Inhibits Oral Squamous Cell Carcinoma Metastasis via Downregulating MCP-1/ CCL2 Expression, J. Biochem, Mol. Toxicol. 29 (2015) 70-76.

[29] S. Raychaudhuri, M. Sinha, D. Mukhopadhyay, N.P. Bhattacharyya, HYPK, a Huntingtin interacting protein, reduces aggregates and apoptosis induced by N- terminal Huntingtin with 40 glutamines in Neuro2a cells and exhibits chaperonelike activity, Hum. Mol. Genet. 17 (2008) 240-255.

[30] A.P. Carter, A.G. Diamant, L. Urnavicius, How dynein and dynactin transport cargos: a structural perspective, Curr. Opin. Struct. Biol. 37 (2016) 62-70.

[31] E.J. Morris, K. Assi, B. Salh, S. Dedhar, Integrin-linked kinase links Dynactin-1/ Dynactin-2 with cortical integrin receptors to orient the mitotic spindle relative to the substratum, Sci. Rep. 5 (2015) 1-11.

[32] P.R. Pär Nordlund, Ribonucleotide reductases, Annu. Rev. Biochem. 75 (2006) 681-706.

[33] K.M. Aird, G. Zhang, H. Li, Z. Tu, B.G. Bitler, A. Garipov, H. Wu, Z. Wei, S.N. Wagner, M. Herlyn, R. Zhang, Suppression of nucleotide metabolism underlies the establishment and maintenance of oncogene-induced senescence, Cell Rep. 3 (2013) 1252-1265.

[34] N. Fatkhutdinov, K. Sproesser, C. Krepler, Q. Liu, P.A. Brafford, M. Herlyn, K.M. Aird, R. Zhang, Targeting RRM2 and mutant BRAF is a novel combinatorial strategy for melanoma, Mol. Cancer Res. 14 (2016) 767-775. 